\title{
Time-resolved dynamics in iodide-uracil-water clusters upon excitation of the nucleobase
}

\author{
Alice Kunin ${ }^{1}$, Valerie S. McGraw ${ }^{1}$, and Daniel M. Neumark ${ }^{1,2, a)}$ \\ ${ }^{1}$ Department of Chemistry, University of California, Berkeley, California 94720, USA. \\ ${ }^{2}$ Chemical Sciences Division, Lawrence Berkeley National Laboratory, Berkeley, California \\ 94720, USA. \\ a)Author to whom correspondence should be addressed. E-mail: dneumark@berkeley.edu.
}

\begin{abstract}
The dynamics of iodide-uracil-water $\left(\mathrm{I}^{-} \cdot \mathrm{U} \cdot \mathrm{H}_{2} \mathrm{O}\right)$ clusters following $\pi-\pi^{*}$ excitation of the nucleobase are probed using time-resolved photoelectron spectroscopy (TRPES).

Photoexcitation of this cluster at $4.77 \mathrm{eV}$ results in electron transfer from the iodide moiety to the uracil, creating a valence-bound (VB) anion within the cross-correlation of the pump and probe laser pulses. This species can decay by a number of channels, including autodetachment and dissociation to $\mathrm{I}^{-}$or larger anion fragments. Comparison of the energetics of the photoexcited cluster and its decay dynamics with those of the bare iodide-uracil $\left(\mathrm{I}^{-} \cdot \mathrm{U}\right)$ complex provide a sensitive probe of the effects of microhydration on these species. The results are also compared with TRPES experiments on $\mathrm{I}^{-} \cdot \mathrm{U} \cdot \mathrm{H}_{2} \mathrm{O}$ at lower pump photon energies, where both the initial photoinduced dynamics and the decay of the photoexcited cluster are qualitatively different.
\end{abstract}




\section{INTRODUCTION}

DNA damage has been shown to proceed directly from UV photoexcitation, as well as indirectly from the attachment of low-energy electrons to its constituent nucleobases. ${ }^{1-4}$ DNA bases exhibit strong absorption cross-sections for UV radiation, particularly near $260 \mathrm{~nm}(4.77$ $\mathrm{eV}),{ }^{5,6}$ and the interaction of nucleobases with surrounding solvent water molecules plays a key role in the relaxation and photostability of nucleobases in this excitation energy regime. ${ }^{7,8}$ The attachment of low-energy electrons has been shown to induce strand breaks in DNA, ${ }^{2-4}$ and it has been proposed that the initial site of electron attachment is the nucleobase followed by electronic coupling that facilitates fragmentation at the backbone. ${ }^{9-15}$ Photoelectron spectroscopy has shown that the addition of water increases the electron affinity of nucleobases, ${ }^{16,17}$ while molecular dynamics simulations have shown that solution-structure fluctuation likely promotes attachment of bulk hydrated electrons to nucleobases. ${ }^{18}$ Our group has previously examined the ultrafast dynamics of electron attachment to nucleobases using time-resolved photoelectron spectroscopy ${ }^{19}\left(\right.$ TRPES) of various iodide-nucleobase $\left(\mathrm{I}^{-} \cdot \mathrm{N}\right)$ complexes $^{20-26}$ and related model systems. $^{27,28}$ The present study uses TRPES to examine iodide-uracil-water $\left(\mathrm{I}^{-} \cdot \mathrm{U} \cdot \mathrm{H}_{2} \mathrm{O}\right)$ clusters photoexcited at $260 \mathrm{~nm}$ and compares these results to those of iodide-uracil $\left(\mathrm{I}^{-} \cdot \mathrm{U}\right)$ to understand the role of water in the mechanisms of excitation and charge transfer in these anionic clusters in this UV excitation regime.

$\mathrm{I}^{-} \cdot \mathrm{N}$ complexes have been previously studied by photofragment action spectroscopy ${ }^{25,29,}$ 30 and electronic structure calculations ${ }^{25,29,31}$ in addition to TRPES. Two regimes of UV photoabsorption have been measured for these complexes. ${ }^{25,29,30}$ The first is centered near the vertical detachment energy (VDE), the difference in energy between the anion and the neutral 
clusters at the equilibrium geometry of the anion, which is approximately $4 \mathrm{eV}$ for $\operatorname{most} \mathrm{I}^{-} \cdot \mathrm{N}$ clusters. $^{20,21,24}$ The second region of UV photoabsorption is broad band spanning $\sim 4.6-5.0 \mathrm{eV}$. Excited state calculations for $\mathrm{I}^{-} \cdot \mathrm{U},{ }^{25}$ iodide-thymine $\left(\mathrm{I}^{-} \cdot \mathrm{T}\right),{ }^{29}$ and $\mathrm{I}^{-} \cdot \mathrm{U} \cdot \mathrm{H}_{2} \mathrm{O}^{31}$ show that photoexcitation near the VDE corresponds to optical excitation from the iodide (5p) orbital to a dipole-bound (DB) state of the nucleobase. The nucleobase DB state arises from capture of the excess electron by the relatively large dipole moment of the base. ${ }^{32-36}$ In contrast, these same calculations indicate that photoexcitation near $4.7-4.8 \mathrm{eV}$ primarily corresponds to basecentered $\pi-\pi^{*}$ excitation.

TRPES of $\mathrm{I}^{-} \cdot \mathrm{N}$ clusters probes the dynamics of photoinduced electron attachment and electronic excitation in nucleobases and traces the time evolution of nascent transient negative ions (TNIs) and anionic decay photofragments. Our previous time-resolved work on $\mathrm{I}^{-} \cdot \mathrm{N}$ binary complexes confirms that the initial dynamics observed in the lower and higher UV photoexcitation regimes are notably different. ${ }^{20-26}$ Near-VDE photoexcitation has been found to rapidly form the DB anion and, with a slight time delay, the valence-bound (VB) anion, as in Eq. 1:

$$
I^{-} \cdot N \stackrel{\mathrm{hv}_{\mathrm{pump}} \approx \operatorname{VDE}\left(\Gamma^{-} \cdot \mathrm{N}\right)}{\longrightarrow}[I \cdots N]_{D B}^{*-},[I \cdots N]_{V B}^{*-}
$$

The VB state corresponds to electron attachment to a valence orbital, the $\pi^{*}$ orbital of the base. ${ }^{14}$, 16,37

Our TRPES studies of $\mathrm{I}^{-} \cdot \mathrm{N}$ clusters with pump excitation energies from $4.60-4.90 \mathrm{eV}$ are expected to create a $\pi-\pi^{*}$ excited state of the nucleobase, as in Eq. 2: 


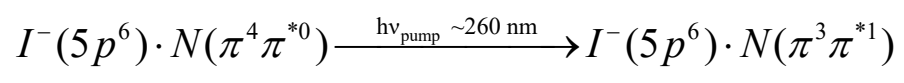

The time-resolved studies in this pump excitation regime have found instantaneous formation of the cluster VB anion with no evidence for the presence of DB states. ${ }^{20,21}$ We have previously $\operatorname{proposed}^{20,26}$ for $\mathrm{I}^{-} \cdot \mathrm{U}$ and $\mathrm{I}^{-} \cdot \mathrm{T}$ complexes that, subsequent to $\pi-\pi^{*}$ excitation of the nucleobase, charge transfer from iodide to the empty $\pi$ orbital creates the VB anion. While this overall mechanism is consistent with experimental results and electronic structure calculations, ${ }^{26}$ it still awaits theoretical confirmation.

Photofragment action spectroscopy in conjunction with TRPES has identified autodetachment as well as formation of $\mathrm{I}^{-}$as the major cluster decay pathways in both photoexcitation regimes for $\mathrm{I}^{-} \cdot \mathrm{U}$ binary clusters, ${ }^{25}$ although the nature of the time-resolved dynamics of these channels has been found to be clearly different for each set of pump energies. ${ }^{26}$ Autodetachment refers to the spontaneous emission of an electron from photoexcitation of an anion resonance embedded within the neutral plus free electron continuum; ${ }^{38,39}$ these electrons can be very slow if randomization of vibrational energy occurs prior to electron emission (the thermionic emission limit). ${ }^{40}$

In the present study, we employ TRPES to excite $\mathrm{I}^{-} \cdot \mathrm{U} \cdot \mathrm{H}_{2} \mathrm{O}$ complexes near the peak of the base-centered $\pi-\pi^{*}$ excitation $^{31}$ at $260 \mathrm{~nm}(4.77 \mathrm{eV})$, and track the resulting dynamics with $1.58 \mathrm{eV}$ or $3.18 \mathrm{eV}$ probe pulses. These experiments, which complement previous work ${ }^{31}$ on near-VDE excitation of $\mathrm{I}^{-} \cdot \mathrm{U} \cdot \mathrm{H}_{2} \mathrm{O}$ complexes, probe the dynamics of both electronically excited uracil as well as the interaction of low-energy electrons with the nucleobase. TRPES can identify and trace the dynamics of nascent TNIs $(\mathrm{eBE} \sim 0 \mathrm{eV}-1 \mathrm{eV})^{17,33,37,41-44}$ with $1.58 \mathrm{eV}$ probe 
pulses, while the higher energy probe is capable of detecting anionic photofragments such as $\mathrm{I}^{-}$ (neutral electron affinity $=3.059 \mathrm{eV}$ ). ${ }^{45}$ Here, we observe formation of the VB anion within the cross-correlation of the pump and probe laser pulses, and subsequent bi-exponential decay in 540 fs and $220 \mathrm{ps}$. Autodetachment and the formation of $\mathrm{I}^{-}$are found to be the major decay channels for the photoexcited clusters. Both the VB anion and the autodetachment signal exhibit long-time decay lifetimes that are an order of magnitude longer than the time-resolved dynamics previously observed for these features in $\mathrm{I}^{-} \cdot \mathrm{U}$ clusters excited at $4.79 \mathrm{eV},{ }^{20,21}$ indicative of the stabilizing effect of water. The $\mathrm{I}^{-}$photofragment is found to form bi-exponentially; we propose that the fast appearance arises from internal conversion of the photoexcited cluster to the anion ground state followed by evaporation of iodide, while the slow appearance may arise from the formation and dissociation of $\mathrm{I}^{-} \cdot \mathrm{H}_{2} \mathrm{O}$.

\section{EXPERIMENTAL METHODS}

The experimental apparatus employed in this study has been described in detail previously ${ }^{46,47}$ and is briefly summarized here. $\mathrm{I}^{-} \cdot \mathrm{U} \cdot \mathrm{H}_{2} \mathrm{O}$ clusters were generated by passing 515 $\mathrm{kPa}$ helium buffer gas over a reservoir of distilled water and a reservoir of methyl iodide $\left(\mathrm{CH}_{3} \mathrm{I}\right)$. The reservoirs and the connecting gas line were wrapped in heating tape; the water reservoir was heated to approximately $30^{\circ} \mathrm{C}$, while the $\mathrm{CH}_{3} \mathrm{I}$ reservoir and gas line were heated to approximately $40{ }^{\circ} \mathrm{C}$. The gas mixture was then passed through an Even-Lavie pulsed valve operating at $500 \mathrm{~Hz}$, which contained a sample cartridge of uracil (Sigma-Aldrich, $\geq 99 \%$ ) heated to $240{ }^{\circ} \mathrm{C}$. The gas mixture was expanded into vacuum through a ring-filament ionizer to create anionic clusters, which were then extracted orthogonally into a Wiley-McLaren time-of-flight 
mass spectrometer. ${ }^{48}$ The $\mathrm{I}^{-} \cdot \mathrm{U} \cdot \mathrm{H}_{2} \mathrm{O}$ clusters were mass-selected and then intersected by the pump and probe laser pulses.

To generate the pump and probe laser pulses, a KMLabs Griffin Oscillator and Dragon Amplifier were used to produce 45 fs pulses centered at approximately $785 \mathrm{~nm}(1.58 \mathrm{eV})$ with $1.8 \mathrm{~mJ} /$ pulse. The fundamental was frequency tripled with two $\beta$-barium borate (BBO) crystals to produce $\sim 10 \mu \mathrm{J} /$ pulse of $260 \mathrm{~nm}(4.77 \mathrm{eV})$ pump pulses. The residual $785 \mathrm{~nm}$ pulses were recovered from the frequency-tripler set-up, and were sent to a delay stage to serve as probe pulses of $\sim 80 \mu \mathrm{J} /$ pulse. Alternatively, this recovered $785 \mathrm{~nm}$ light was frequency-doubled in a BBO to produce $\sim 60 \mu \mathrm{J} /$ pulse of $390 \mathrm{~nm}(3.18 \mathrm{eV})$ probe pulses. In either case, the pump and probe pulses were combined at the chamber in a dichroic beamsplitter. The cross-correlation of $260 \mathrm{~nm} / 785 \mathrm{~nm}$ was $\leq 180 \mathrm{fs}$ and that of $260 \mathrm{~nm} / 390 \mathrm{~nm}$ was $<300 \mathrm{fs}$; the latter cross-correlation, measured outside the chamber, is obscured by residual $390 \mathrm{~nm}$ light, and the actual crosscorrelation is expected to be as much as 50-100 fs shorter than this measured value. ${ }^{25}$

The resultant photoelectrons were analyzed by a velocity-map imaging assembly ${ }^{49}$ comprising three electron optical elements and a chevron-stacked microchannel plate detector coupled to a phosphor screen imaged by a charge-coupled device camera. Basis-set expansion (BASEX) reconstruction methods were used to reconstruct the 3D photoelectron kinetic energy (eKE) distributions. ${ }^{50}$

\section{RESULTS}

Fig. 1 presents a laser noise-subtracted, single-photon photoelectron spectrum of $\mathrm{I}^{-} \cdot \mathrm{U} \cdot \mathrm{H}_{2} \mathrm{O}$ clusters photodetached at $4.74 \mathrm{eV} \cdot{ }^{31}$ This spectrum is plotted as a function of electron binding energy $(\mathrm{eBE})\left(\mathrm{eBE}=\mathrm{h} v_{\text {photon }}-\mathrm{eKE}\right)$. Two features appear in this spectrum: feature A, 
peaked at approximately $4.69 \pm 0.05 \mathrm{eV}$ eBE (or $0.05 \pm 0.05 \mathrm{eV}$ eKE), and feature $\mathrm{B}$, peaked at $4.40 \pm 0.05 \mathrm{eV}$ eBE (or $0.37 \pm 0.05 \mathrm{eV} \mathrm{eKE}$ ). As has been previously determined, ${ }^{31}$ feature A corresponds to autodetachment of $\sim 0 \mathrm{eV}$ eKE electrons from the photoexcited $\mathrm{I}^{-} \cdot \mathrm{U} \cdot \mathrm{H}_{2} \mathrm{O}$ clusters, and feature $\mathrm{B}$ corresponds to direct detachment to the lower iodine spin-orbit state $\left({ }^{2} \mathrm{P}_{3 / 2}\right)$ from the $\mathrm{I}^{-} \cdot \mathrm{U} \cdot \mathrm{H}_{2} \mathrm{O}$ anion, yielding a VDE of $4.40 \mathrm{eV}$ for $\mathrm{I}^{-} \cdot \mathrm{U} \cdot \mathrm{H}_{2} \mathrm{O}$. As both of these features arise from single-photon (pump-only) processes at the pump energy employed in our TRPES experiments, they are also present in all of the TRPE spectra here at these same eKEs.

Fig. 2 presents photoelectron spectra at select time delays for $\mathrm{I}^{-} \cdot \mathrm{U} \cdot \mathrm{H}_{2} \mathrm{O}$ at $4.77 \mathrm{eV}$ pump excitation energy and $1.58 \mathrm{eV}$ probe energy; here and in other TRPE spectra, $\mathrm{eBE}=\mathrm{h} v_{\text {probe }}-$ eKE. Feature A exhibits non-zero intensity at negative times and increases in intensity over approximately 10 ps before decreasing back to its initial intensity. Feature B exhibits noisy but similar time dynamics as feature A, likely due to spectral overlap between the two features in this region. Feature $\mathrm{C}$, covering the region from approximately $0.1 \mathrm{eV}-0.9 \mathrm{eV} \mathrm{eBE}$, is enlarged in the inset. Based on our previous results on $\mathrm{I}^{-} \cdot \mathrm{U}$ clusters photoexcited at excitation energies in the range of $4.69-4.90 \mathrm{eV},{ }^{20,21}$ we can assign feature $\mathrm{C}$ as the $\mathrm{VB}$ anion of photoexcited $\mathrm{I}^{-} \cdot \mathrm{U} \cdot \mathrm{H}_{2} \mathrm{O}$. Time-resolved photoelectron spectra for feature $\mathrm{C}$, background-subtracted with respect to the most negative delay time, are shown in Fig. 3 for time delays up to 5 ps.

Fig. 4 presents photoelectron spectra at select time delays for $\mathrm{I}^{-} \cdot \mathrm{U} \cdot \mathrm{H}_{2} \mathrm{O}$ at $4.77 \mathrm{eV}$ pump excitation energy and $3.18 \mathrm{eV}$ probe energy. Features A and B are the same features seen in Fig. 2. The prominent new feature in these spectra, feature $\mathrm{D}$, is located at $3.06 \pm 0.05 \mathrm{eV}$ eBE, and this narrow feature grows monotonically in intensity over the course of experiment. Based on the binding energy, spectral shape, and time-dynamics of feature $\mathrm{D}$, we assign feature $\mathrm{D}$ to 
photodetachment of atomic iodide to the ${ }^{2} \mathrm{P}_{3 / 2}$ iodine spin-orbit state. Background-subtracted time-resolved photoelectron spectra for features A and D are presented in Fig. 5.

\section{ANALYSIS}

The normalized, integrated intensities for the VB anion (red, feature C) and the autodetachment feature (black, feature A) are shown at early, intermediate, and long time delays in Fig. 5. The integrated signals are fit to the convolution of a Gaussian instrumental response function with $i$ exponential functions as in Eq. 3:

$$
I(t)=\frac{1}{\sigma_{C C} \sqrt{2 \pi}} \exp \left(\frac{-t^{2}}{2 \sigma_{C C}^{2}}\right) \cdot\left\{\begin{array}{c}
I_{0}, t<0 \\
I_{0}+\sum_{i} A_{i} \exp \left(\frac{-t}{\tau_{i}}\right), t \geq 0
\end{array}\right.
$$

In this equation, $\sigma_{c c}$ is the Gaussian full width at the half-maximum given by the crosscorrelation of the pump and probe laser pulses, $I_{0}$ is the signal background, $A_{i}$ are the coefficients for each exponential function, and $\tau_{i}$ are the corresponding rise or decay lifetimes for each exponential. These fits are shown in Fig. 5 as solid red and black lines for the VB anion and autodetachment features, respectively. The VB anion is found to appear within the crosscorrelation of the pump and probe laser pulses and decay bi-exponentially. The fits for the $\mathrm{I}^{-} \cdot \mathrm{U} \cdot \mathrm{H}_{2} \mathrm{O}$ VB anion decay are $540 \pm 240 \mathrm{fs}$ and $220 \pm 70 \mathrm{ps}$. The autodetachment feature was found to remain relatively constant in intensity at negative times. At positive delay times this feature rises to a maximum in $11.3 \pm 2.2 \mathrm{ps}$ followed by decay to the negative-time intensity in approximately $285 \pm 70 \mathrm{ps}$. 
For ease of comparison to our previous work on $\mathrm{I}^{-} \cdot \mathrm{U}$ clusters, the normalized, integrated intensities for the VB anion and autodetachment produced from $\mathrm{I}^{-} \cdot \mathrm{U}$ photoexcited at $4.79 \mathrm{eV}$ are presented in Fig. 7 in purple, overlaid with the corresponding $\mathrm{I}^{-} \cdot \mathrm{U} \cdot \mathrm{H}_{2} \mathrm{O}$ features (green) from Fig. 5. Fig. 8 presents the normalized integrated intensity and fitted rise for the $\mathrm{I}^{-}$signal observed here. The $\mathrm{I}^{-}$formation was found to be bi-exponential, with rise time constants of $32.5 \pm 2.6 \mathrm{ps}$ and $230 \pm 20$ ps. Table I summarizes the fit rise and decay lifetimes for the VB anion, autodetachment, and $\mathrm{I}^{-}$feature for $\mathrm{I}^{-} \cdot \mathrm{U} \cdot \mathrm{H}_{2} \mathrm{O}$ in the present study and for $\mathrm{I}^{-} \cdot \mathrm{U}$ photoexcited at $4.79 \mathrm{eV}$ from our past work. ${ }^{20,25}$

\section{DISCUSSION}

This work explores the dynamics of $\mathrm{I}^{-} \cdot \mathrm{U} \cdot \mathrm{H}_{2} \mathrm{O}$ clusters photoexcited at $4.77 \mathrm{eV}$, resonant with the base-centered $\pi-\pi^{*}$ transition for uracil. We observe instantaneous formation of the VB anion of the cluster following photoexcitation, and bi-exponential decay of this species with time constants of $540 \mathrm{fs}$ and $220 \mathrm{ps}$. Autodetachment and $\mathrm{I}^{-}$re-formation are observed as decay channels in this photoexcitation regime. These experiments show that, to first order, the dynamics of photoexcited $\mathrm{I}^{-} \cdot \mathrm{U} \cdot \mathrm{H}_{2} \mathrm{O}$ are similar to those of $\mathrm{I}^{-} \cdot \mathrm{U}$ in both the near-VDE and $\pi-\pi^{*}$ excitation regimes. In both species, near-VDE excitation results in DB state formation and slightly delayed VB state formation attributed to partial $\mathrm{DB} \rightarrow \mathrm{VB}$ conversion, whereas excitation of the higher energy band results in VB formation only within the cross-correlation of the laser pulses. However, the addition of a water molecule noticeably affects the decay dynamics of the VB state and the rise time of the $\mathrm{I}^{-}$signal, as can be seen from Fig. 6 and Table I. In this section, we consider the overall photoexcitation mechanism and the effect of the added water on the longer time dynamics in more detail. 
We have previously proposed that excitation of $\mathrm{I}^{-} \cdot \mathrm{U}$ and $\mathrm{I}^{-} \cdot \mathrm{T}$ in this energy regime is dominated by the base-centered $\pi-\pi^{*}$ transition, ${ }^{26}$ and our excited state calculations for the $\mathrm{I}^{-} \cdot \mathrm{U} \cdot \mathrm{H}_{2} \mathrm{O}$ complex ${ }^{31}$ indicate that this is expected to be the case here as well. These calculations find no oscillator strength for a direct optical transition to form the VB anion via electron transfer from the $\mathrm{I}^{-}$, thus we have proposed that following $\pi-\pi^{*}$ photoexcitation, iodide rapidly transfers an electron to fill the hole in the $\pi$ orbital. ${ }^{26}$ The instantaneous appearance of the VB anion in the present work is congruent with the past TRPES results of $\mathrm{I}^{-} \cdot \mathrm{U}$ and $\mathrm{I}^{-} \cdot \mathrm{T}$ in this pump excitation regime, and indicates that the same mechanism for the formation of the VB anion is expected to be operative here.

As shown in Eq. 4 below, we have previously proposed that the $\pi$ - $\pi$ * iodide-associated photoexcited state decays by two pathways: iodide transfers a valence electron to fill the hole in the nucleobase $\pi$ orbital, creating a VB anion that then decays by autodetachment, or the $\pi-\pi^{*}$ excited nucleobase state internally converts to the ground state of the cluster and subsequently evaporates iodide. $^{26}$

$$
\begin{aligned}
I^{-}\left(5 p^{6}\right) \cdot N\left(\pi^{4} \pi^{* 0}\right) & \stackrel{\mathrm{hv}_{\text {pump }} \sim 260 \mathrm{~nm}}{\longrightarrow} I^{-}\left(5 p^{6}\right) \cdot N\left(\pi^{3} \pi^{* 1}\right) \\
I^{-} \cdot N\left(\pi^{3} \pi^{* 1}\right) & \rightarrow I \cdot N^{-}\left(\pi^{4} \pi^{* 1}\right) \stackrel{\text { Autodetachment }}{\longrightarrow} I \cdot N+\mathrm{e}^{-} \\
& \stackrel{\substack{\text { Internal } \\
\text { Conversion }}}{\longrightarrow} I^{-} \cdot N \rightarrow I^{-}+N
\end{aligned}
$$

The present TRPES experiment of $\mathrm{I}^{-} \cdot \mathrm{U} \cdot \mathrm{H}_{2} \mathrm{O}$ is sensitive to each of these decay channels in Eq. 4. In the subsections that follow, we consider the VB anion, autodetachment, and iodide formation dynamics and explore possible mechanisms for the observed behavior that expand on the basic framework previously set forward. 
A. VB anion formation and decay dynamics

As seen in Fig. 3, the VB anion of $\mathrm{I}^{-} \cdot \mathrm{U} \cdot \mathrm{H}_{2} \mathrm{O}$ appears to have the strongest intensity near $0.8-0.9 \mathrm{eV}$ eBE, which is approximately $0.2-0.3 \mathrm{eV}$ higher than the peak eBE for the VB anion of $\mathrm{I}^{-} \cdot \mathrm{U}$ in this excitation energy regime. ${ }^{20,21}$ This finding indicates that the water molecule is attached in the VB anion complex and that the presence of water stabilizes the VB anion. Increased TNI binding energies upon addition of water were previously observed following nearVDE photoexcitation of $\mathrm{I}^{-} \cdot \mathrm{U} \cdot \mathrm{H}_{2} \mathrm{O}$ as compared to the TNIs of near-VDE photoexcited $\mathrm{I}^{-} \cdot \mathrm{U}$ clusters. ${ }^{31}$ This energy increase of $0.2-0.3 \mathrm{eV}$ eBE is similar to the stabilization of the TNIs in the near-VDE photoexcitation regime, although we note that in both cases the presence of feature B, single-photon detachment to $\left.\mathrm{I}^{2} \mathrm{P}_{3 / 2}\right) \cdot \mathrm{U}\left(\cdot \mathrm{H}_{2} \mathrm{O}\right)$, may obscure the full spectral width of the VB state.

In the present study, the VB anion is found to appear within the cross-correlation of the pump and probe laser pulses and decay bi-exponentially, as is the case for $\mathrm{I}^{-} \cdot \mathrm{U}$ photoexcited in this excitation energy regime. The fast decay of the $\mathrm{VB}$ anion is similar to that of the $\mathrm{I} \cdot \mathrm{U}^{-} \mathrm{VB}$ anion formed at $4.79 \mathrm{eV}$ pump energy (Table I), while the long decay of the VB anion is an order of magnitude longer in the water-associated complex. This long-lived VB anion stabilization is commensurate with the dynamics that were previously observed in near-VDE photoexcited $\mathrm{I}^{-} \cdot \mathrm{U} \cdot \mathrm{H}_{2} \mathrm{O}$ clusters, in which the VB anion long-time decay by autodetachment was found to be significantly longer with the addition of water. ${ }^{31}$

In our previous results for $\pi-\pi^{*}$ photoexcited $\mathrm{I}^{-} \cdot \mathrm{U}$ and $\mathrm{I}^{-} \cdot \mathrm{T}$ clusters, we proposed that the VB anion decays by autodetachment because the measured autodetachment signals for both clusters were found to exhibit prompt depletion and recovery near $t_{0}$ that mirrored the appearance and fast decay of the VB anion at early times, although the $\mathrm{I}^{-} \cdot \mathrm{U}$ VB anion exhibited a somewhat 
longer-lived bi-exponential decay. ${ }^{21,26}$ Given the similarities between the $\mathrm{I}^{-} \cdot \mathrm{U}$ and $\mathrm{I}^{-} \cdot \mathrm{U} \cdot \mathrm{H}_{2} \mathrm{O}$ complexes, it is likely that the bi-exponential decay mechanism for the $\mathrm{I}^{-} \cdot \mathrm{U} \cdot \mathrm{H}_{2} \mathrm{O}$ VB anion is by autodetachment as well. Previously, we have attributed bi-exponential decay of the VB anion in this pump energy regime to the loss of neutral iodine from the cluster, causing a reduction in the internal energy and resulting in two autodetachment decay components. ${ }^{23}$ Neutral iodine loss was implicated in the VDE shifting dynamics of the DB anion observed in near-VDE photoexcited $\mathrm{I}^{-} \cdot \mathrm{U}, \mathrm{I}^{-} \cdot \mathrm{T}$, and $\mathrm{I}^{-} \cdot \mathrm{U} \cdot \mathrm{H}_{2} \mathrm{O}$ complexes. ${ }^{22,23,31} \mathrm{In}$ the $\pi-\pi^{*}$ photoexcitation regime, the $\mathrm{I} \cdot \mathrm{T}^{-} \mathrm{VB}$ anion exhibits mono-exponential decay dynamics, and it has been suggested that the initial autodetachment may be faster compared to iodine loss than in $\mathrm{I}^{-} \cdot \mathrm{U}^{23}$ The $\mathrm{I}^{-} \cdot \mathrm{U} \cdot \mathrm{H}_{2} \mathrm{O}$ results here further indicate that the bi-exponential or mono-exponential nature of the VB anion decay is sensitive to the electronic structure of the specific nucleobase species.

\section{B. Autodetachment dynamics}

First, we begin by commenting on the nature of the time-resolved autodetachment signals observed in our TRPES studies. Fig. 1 shows that autodetachment signal occurs in the $4.74 \mathrm{eV}$ single-photon PES of $\mathrm{I}^{-} \cdot \mathrm{U} \cdot \mathrm{H}_{2} \mathrm{O},{ }^{31}$ and therefore clearly arises from a one-photon (pump-only) process. As seen in Figs. 2 and 4, the autodetachment signal exhibits considerable intensity at all times, including negative times where the probe pulse precedes the pump pulse. Therefore, the normalized, time-resolved signals in Fig. 6 reflect that the autodetachment intensity exhibits little to no decrease below its intensity level at negative times. At positive probe pulse arrival times, the autodetachment signal is found to rise in intensity within $11.3 \mathrm{ps}$ and then decay in approximately $285 \mathrm{ps}$. It is important to note that our experiment does not directly measure timeresolved autodetachment dynamics since the autodetachment electron signal is generated 
spontaneously and not by the probe pulse. Instead, the observed time-dependent integrated intensities associated with autodetachment signal arise from probe-based interactions that affect the amount of autodetachment signal that is detected for a given probe arrival time.

Our work on both $\mathrm{I}^{-} \cdot \mathrm{U} \cdot \mathrm{H}_{2} \mathrm{O}$ and $\mathrm{I}^{-} \cdot \mathrm{U}$ clusters has measured autodetachment arising from near-VDE photoexcitation, ${ }^{22,31}$ as well as from photoexcitation in the region of the base-centered $\pi-\pi^{*}$ transition (shown for both $\mathrm{I}^{-} \cdot \mathrm{U} \cdot \mathrm{H}_{2} \mathrm{O}$ and $\mathrm{I}^{-} \cdot \mathrm{U}$ in Fig. $7 \mathrm{c}$ ). ${ }^{20,21,25}$ Each of these past studies, regardless of photoexcitation energy, has shown autodetachment that exhibits depletion at $t_{0}$ followed by recovery to or beyond its initial intensity. Near-VDE photoexcitation in both clusters yields autodetachment signal that appears to at least qualitatively mirror the respective TNI dynamics; an example for $\mathrm{I}^{-} \cdot \mathrm{U} \cdot \mathrm{H}_{2} \mathrm{O}$ near-VDE pump autodetachment signal is shown in Fig. S1. Concomitant autodetachment signal depletion and recovery at $t_{0}$ with time constants that mirror the TNI appearance and fast decay indicates that the probe pulse at early times photodetaches the nascent TNI population that would otherwise decay to produce autodetachment signal in the absence of the probe. At later times, the probe laser interacts with a decreased population of anions that have not already undergone autodetachment, so one expects less depletion of the autodetachment signal and eventually no depletion at all. Under these circumstances, the recovery of the autodetachment signal yields the lifetime of the autodetaching state. ${ }^{51}$

In Fig. $7 \mathrm{c}$, it can be seen that $\pi-\pi^{*}$ photoexcitation for both $\mathrm{I}^{-} \cdot \mathrm{U} \cdot \mathrm{H}_{2} \mathrm{O}$ and $\mathrm{I}^{-} \cdot \mathrm{U}$ clusters yields an unexpected "overshoot" of the autodetachment intensity beyond the negative-time intensity in $10 \mathrm{~s}$ of ps. The autodetachment intensity overshoot, therefore, is uniquely associated with the VB anion generated following $\pi-\pi^{*}$ photoexcitation. Moreover, the similar autodetachment dynamics for $\mathrm{I}^{-} \cdot \mathrm{U}$ and $\mathrm{I}^{-} \cdot \mathrm{U} \cdot \mathrm{H}_{2} \mathrm{O}$ in contrast to those of $\pi-\pi^{*}$ photoexcited $\mathrm{I}^{-} \cdot \mathrm{T}$ 
clusters, which do not show any autodetachment intensity overshoot, ${ }^{21}$ suggest that the overshoot is also nucleobase-specific and likely related to the presence of the long-lived VB state.

This overshoot may arise if the probe pulse is absorbed by the VB anion and excites the TNI to a higher-lying excited state that subsequently decays by autodetachment, as in Eq. 5:

$$
\begin{aligned}
& I^{-} \cdot U\left(\pi^{4} \pi^{* 0}\right) \cdot H_{2} O \stackrel{\text { hv pump }_{\text {pur }}}{\longrightarrow} I^{-} \cdot U\left(\pi^{3} \pi^{* 1}\right) \cdot H_{2} O \rightarrow I \cdot U^{-}\left(\pi^{4} \pi^{* 1}\right) \cdot H_{2} O \\
& I \cdot U^{-}\left(\pi^{4} \pi^{* 1}\right) \cdot H_{2} O \stackrel{\text { Autodetachment }}{\longrightarrow} I \cdot U \cdot H_{2} O+\mathrm{e}^{-} \\
& \stackrel{\text { hv }_{\text {probe }}}{\longrightarrow}\left[I \cdot U^{-}\left(\pi^{4} \pi^{* 1}\right) \cdot H_{2} O\right]^{*} \stackrel{\text { Autodetachment }}{\longrightarrow} I \cdot U \cdot H_{2} O+\mathrm{e}^{-}
\end{aligned}
$$

The autodetachment intensity overshoot is only observed in $\mathrm{I}^{-} \cdot \mathrm{U}$ and $\mathrm{I}^{-} \cdot \mathrm{U} \cdot \mathrm{H}_{2} \mathrm{O}$ clusters, which both exhibit longer-lived, bi-exponentially decaying VB anions. $\mathrm{I}^{-} \cdot \mathrm{T}$ clusters in this pump energy region, in contrast, do not exhibit autodetachment intensity overshoot, and the VB anion decays mono-exponentially in only $\sim 500 \mathrm{fs} .{ }^{23}$ As seen in Table I, the long decay lifetimes for the VB anions of $\mathrm{I}^{-} \cdot \mathrm{U}$ and $\mathrm{I}^{-} \cdot \mathrm{U} \cdot \mathrm{H}_{2} \mathrm{O}$ clusters are in agreement with the decay lifetimes of the excess autodetachment signal. Thus, we believe a mechanism as in Eq. 5 is operative here for both $\mathrm{I}^{-} \cdot \mathrm{U}$ and $\mathrm{I}^{-} \cdot \mathrm{U} \cdot \mathrm{H}_{2} \mathrm{O}$ photoexcited in this pump energy regime.

The presence of water appears to somewhat slow the rise of the autodetachment feature (Table I), and, notably, the autodetachment signal decay lifetime is an order of magnitude longer for $\mathrm{I}^{-} \cdot \mathrm{U} \cdot \mathrm{H}_{2} \mathrm{O}$ than $\mathrm{I}^{-} \cdot \mathrm{U}$. As has been observed for the $\mathrm{I}^{-} \cdot \mathrm{U} \cdot \mathrm{H}_{2} \mathrm{O}$ VB anion both in the $\pi-\pi^{*}$ pump energy regime as well as the near-VDE excitation regime, the interaction of water may stabilize the excited cluster to decay by autodetachment, increasing the observed lifetime. The autodetachment signal resulting from $\mathrm{I}^{-} \cdot \mathrm{U} \cdot \mathrm{H}_{2} \mathrm{O}$ also does not appear to have depletion near $\mathrm{t}_{0}$ as was observed in both $\mathrm{I}^{-} \cdot \mathrm{U}$ and $\mathrm{I}^{-} \cdot \mathrm{T}$ clusters. ${ }^{20,21}$ As noted earlier, the autodetachment depletion 
at early probe arrival times arises due to photodetachment of the VB excited state, which would otherwise be the spontaneous source of autodetached electrons. Lack of notable depletion in the early-time autodetachment signal of $\mathrm{I}^{-} \cdot \mathrm{U} \cdot \mathrm{H}_{2} \mathrm{O}$ as compared to $\mathrm{I}^{-} \cdot \mathrm{U}$ may therefore indicate that the $\mathrm{I}^{-} \cdot \mathrm{U} \cdot \mathrm{H}_{2} \mathrm{O}$ VB anion is more strongly stabilized relative to autodetachment than $\mathrm{I}^{-} \cdot \mathrm{U}$ clusters in this photoexcitation regime. Finally, it is interesting to note that the observed autodetachment dynamics in $\pi-\pi^{*}$ photoexcited $\mathrm{I}^{-} \cdot \mathrm{U} \cdot \mathrm{H}_{2} \mathrm{O}$ clusters are approximately the same in both the 1.58 $\mathrm{eV}$ and $3.18 \mathrm{eV}$ probe energy studies (Fig. S2). It is possible, given the relatively high power of the $1.58 \mathrm{eV}$ probe pulses employed in the present study, that the $1.58 \mathrm{eV}$ autodetachment dynamics arise from the absorption of two probe photons by the VB anion, particularly if absorption of the first photon is resonant. This would yield similar autodetachment overshoot dynamics for each probe energy.

C. Iodide formation dynamics

TRPES of $\pi-\pi^{*}$ photoexcited $\mathrm{I}^{-} \cdot \mathrm{U} \cdot \mathrm{H}_{2} \mathrm{O}$ clusters finds bi-exponential $\mathrm{I}^{-}$formation in 32.5 ps and $230 \mathrm{ps}$, in contrast to $\mathrm{I}^{-} \cdot \mathrm{U}$ clusters for which $\mathrm{I}^{-}$was found to appear mono-exponentially in $36 \mathrm{ps}^{25}$ Both $\mathrm{I}^{-} \cdot \mathrm{U} \cdot \mathrm{H}_{2} \mathrm{O}$ and $\mathrm{I}^{-} \cdot \mathrm{U}$ clusters yield bi-exponential $\mathrm{I}^{-}$formation following nearVDE photoexcitation, ${ }^{25,31}$ and we have attributed the bi-exponential rise dynamics in both cases to internal conversion followed by iodide evaporation from both the DB and VB anions that are formed in this pump energy regime. We have previously proposed, due to the strong connection between the VB anion in this pump energy regime and the autodetachment dynamics, ${ }^{26}$ that base-centered $\pi-\pi^{*}$ excitation is followed by internal conversion to the ground state and subsequent dissociation to produce iodide mono-exponentially, as in Eq. 4. While this 
mechanism is likely the source of the fast $\mathrm{I}^{-}$rise signal, it alone cannot fully explain the origin of the bi-exponential $\mathrm{I}^{-}$appearance observed here for $\mathrm{I}^{-} \cdot \mathrm{U} \cdot \mathrm{H}_{2} \mathrm{O}$ clusters.

Several energetically accessible dissociation channels have been previously calculated for $\mathrm{I}^{-} \cdot \mathrm{U} \cdot \mathrm{H}_{2} \mathrm{O}$ clusters, including dissociation to yield $\mathrm{I}^{-} \cdot \mathrm{H}_{2} \mathrm{O}$ clusters. ${ }^{31} \mathrm{I}^{-} \cdot \mathrm{H}_{2} \mathrm{O}$ produced as a dissociation product upon photoexcitation could also then further dissociate to yield a second source of $\mathrm{I}^{-}$signal with a delayed rise time, contributing to the bi-exponential rise dynamics observed here, as depicted in Eq. 6:

$$
\begin{aligned}
& I^{-} \cdot U \cdot \mathrm{H}_{2} \mathrm{O} \stackrel{\mathrm{hv}_{\text {pump }} 2260 \mathrm{~nm}}{\longrightarrow} I^{-} \cdot U\left(\pi^{3} \pi^{* 1}\right) \cdot \mathrm{H}_{2} \mathrm{O} \\
& I^{-} \cdot U\left(\pi^{3} \pi^{* 1}\right) \cdot \mathrm{H}_{2} \mathrm{O} \stackrel{\text { Internal }}{\text { Conversion }} \longrightarrow I^{-} \cdot U \cdot \mathrm{H}_{2} \mathrm{O} \rightarrow I^{-}+U \cdot \mathrm{H}_{2} \mathrm{O} \\
& \rightarrow I^{-} \cdot \mathrm{H}_{2} \mathrm{O}+U \rightarrow I^{-}+\mathrm{H}_{2} \mathrm{O}+U
\end{aligned}
$$

TRPES experiments of $\mathrm{I}^{-} \cdot \mathrm{U} \cdot \mathrm{H}_{2} \mathrm{O}$ clusters photoexcited at $4.77 \mathrm{eV}$ pump energy and probed by $4.0 \mathrm{eV}$ and by $4.77 \mathrm{eV}$ probe energies were unable to conclusively identify the formation of $\mathrm{I}^{-} \cdot \mathrm{H}_{2} \mathrm{O}(\mathrm{VDE}=3.51 \pm 0.02 \mathrm{eV})^{52,53}$ due to poor overall signal levels at these probe energies. However, the existence of this dissociation channel could be verified in future experiments by photofragment action spectroscopy, for example.

\section{CONCLUSIONS}

TRPES has been used to examine TNI formation and photodissociation in $\pi$ - $\pi$ * photoexcited $\mathrm{I}^{-} \cdot \mathrm{U} \cdot \mathrm{H}_{2} \mathrm{O}$ clusters. We observe instantaneous formation of the VB anion of the complex, and propose that this state arises from charge transfer from iodide to fill the empty $\pi$ orbital that remains on uracil after the nucleobase is photoexcited, consistent with the proposed 
mechanism for $\mathrm{I}^{-} \cdot \mathrm{U}$ binary clusters. The VB anion of $\mathrm{I}^{-} \cdot \mathrm{U} \cdot \mathrm{H}_{2} \mathrm{O}$ exhibits increased binding energy compared to the $\mathrm{VB}$ anion of $\mathrm{I}^{-} \cdot \mathrm{U}$ clusters in this photoexcitation regime. The $\mathrm{VB}$ anion is found to decay bi-exponentially, as in $\mathrm{I}^{-} \cdot \mathrm{U}$, but the long decay lifetime is an order of magnitude longer in $\mathrm{I}^{-} \cdot \mathrm{U} \cdot \mathrm{H}_{2} \mathrm{O}$ clusters and is expected to reflect stabilization of the VB state relative to decay by autodetachment. Autodetachment and the formation of $\mathrm{I}^{-}$are measured as dissociation channels of the photoexcited $\mathrm{I}^{-} \cdot \mathrm{U} \cdot \mathrm{H}_{2} \mathrm{O}$ complexes. Overshoot of the measured autodetachment intensity beyond the negative-time autodetachment signal indicates that absorption of the probe pulse by the long-lived VB anion may produce additional autodetachment intensity. Bi-exponential formation of $\mathrm{I}^{-}$is found in $32.5 \mathrm{ps}$ and $230 \mathrm{ps}$, and we predict the unique bi-exponential rise dynamics measured in $\mathrm{I}^{-} \cdot \mathrm{U} \cdot \mathrm{H}_{2} \mathrm{O}$ clusters in this photoexcitation regime are the result of additional dissociation channels that arise from the presence of water. Internal conversion of the $\pi-\pi^{*}$ excited state and subsequent cluster dissociation is expected to yield $\mathrm{I}^{-}$, with long-time rising signal that may be produced by dissociation of $\pi-\pi^{*}$ photoexcited $\mathrm{I}^{-} \cdot \mathrm{U} \cdot \mathrm{H}_{2} \mathrm{O}$ to yield $\mathrm{I}^{-} \cdot \mathrm{H}_{2} \mathrm{O}$ that can subsequently decay to yield iodide.

\section{SUPPLEMENTARY MATERIAL}

See supplementary material for additional figures of the normalized integrated intensity for the autodetachment signal arising from $\mathrm{I}^{-} \cdot \mathrm{U} \cdot \mathrm{H}_{2} \mathrm{O}$ clusters at $4.38 \mathrm{eV}$ pump excitation energy and $3.14 \mathrm{eV}$ probe energy, as well as a comparison of the autodetachment signal arising from $\mathrm{I}^{-} \cdot \mathrm{U} \cdot \mathrm{H}_{2} \mathrm{O}$ clusters at $4.77 \mathrm{eV}$ pump excitation energy and $1.58 \mathrm{eV}$ probe energy vs. $3.18 \mathrm{eV}$ probe energy. 


\section{ACKNOWLEDGEMENTS}

This research was funded by the National Science Foundation under Grant No. CHE1663832. V.S.M. gratefully acknowledges support from a National Science Foundation Graduate Research Fellowship.

\section{References}

${ }^{1}$ J. Kohanoff, M. McAllister, G. A. Tribello, and B. Gu, J. Phys. - Condens. Mat. 29, 383001 (2017).

${ }^{2}$ B. Boudaiffa, P. Cloutier, D. Hunting, M. A. Huels, and L. Sanche, Science 287, 1658 (2000).

${ }^{3}$ F. Martin, P. D. Burrow, Z. Cai, P. Cloutier, D. Hunting, and L. Sanche, Phys. Rev. Lett. 93, 068101 (2004).

${ }^{4}$ E. Alizadeh, and L. Sanche, Chem. Rev. 112, 5578 (2012).

${ }^{5}$ D. Voet, W. B. Gratzer, R. A. Cox, and P. Doty, Biopolymers 1, 193 (1963).

${ }^{6}$ T. Gustavsson, Á. Bányász, E. Lazzarotto, D. Markovitsi, G. Scalmani, M. J. Frisch, V. Barone, and R. Improta, J. Am. Chem. Soc. 128, 607 (2006).

${ }^{7}$ Y. He, C. Wu, and W. Kong, J. Phys. Chem. A 107, 5145 (2003).

${ }^{8}$ Y. He, C. Wu, and W. Kong, J. Phys. Chem. A 108, 943 (2004).

${ }^{9}$ R. Barrios, P. Skurski, and J. Simons, J. Phys. Chem. B 106, 7991 (2002).

${ }^{10}$ G. Hanel, B. Gstir, S. Denifl, P. Scheier, M. Probst, B. Farizon, M. Farizon, E. Illenberger, and T. D. Märk, Phys. Rev. Lett. 90, 188104 (2003).

${ }^{11}$ J. Berdys, I. Anusiewicz, P. Skurski, and J. Simons, J. Am. Chem. Soc. 126, 6441 (2004).

${ }^{12}$ J. Berdys, P. Skurski, and J. Simons, J. Phys. Chem. B 108, 5800 (2004).

${ }^{13}$ S. Ptasińska, S. Denifl, S. Gohlke, P. Scheier, E. Illenberger, and T. D. Märk, Angew. Chem. Int. Ed. 45, 1893 (2006).

${ }^{14}$ J. Simons, Accounts Chem. Res. 39, 772 (2006).

${ }^{15}$ H.-Y. Chen, P.-Y. Yang, H.-F. Chen, C.-L. Kao, and L.-W. Liao, J. Phys. Chem. B 118, 11137 (2014).

${ }^{16}$ J. H. Hendricks, S. A. Lyapustina, H. L. de Clercq, and K. H. Bowen, J. Chem. Phys. 108, 8 (1998). 
${ }^{17}$ J. Schiedt, R. Weinkauf, D. M. Neumark, and E. W. Schlag, Chem. Phys. 239, 511 (1998).

${ }^{18}$ J. Zhao, M. Wang, A. Fu, H. Yang, and Y. Bu, ChemPhysChem 16, 2348 (2015).

${ }^{19}$ A. Stolow, A. E. Bragg, and D. M. Neumark, Chem. Rev. 104, 1719 (2004).

${ }^{20}$ M. A. Yandell, S. B. King, and D. M. Neumark, J. Am. Chem. Soc. 135, 2128 (2013).

${ }^{21}$ S. B. King, M. A. Yandell, and D. M. Neumark, Faraday Discuss. 163, 59 (2013).

${ }^{22}$ S. B. King, M. A. Yandell, A. B. Stephansen, and D. M. Neumark, J. Chem. Phys. 141, 224310 (2014).

${ }^{23}$ S. B. King, A. B. Stephansen, Y. Yokoi, M. A. Yandell, A. Kunin, T. Takayanagi, and D. M. Neumark, J. Chem. Phys. 143, 024312 (2015).

${ }^{24}$ A. B. Stephansen, S. B. King, Y. Yokoi, Y. Minoshima, W.-L. Li, A. Kunin, T. Takayanagi, and D. M. Neumark, J. Chem. Phys. 143, 104308 (2015).

${ }^{25}$ W.-L. Li, A. Kunin, E. Matthews, N. Yoshikawa, C. E. H. Dessent, and D. M. Neumark, J. Chem. Phys. 145, 044319 (2016).

${ }^{26}$ A. Kunin, and D. M. Neumark, Phys. Chem. Chem. Phys. 21, 7239 (2019).

${ }^{27}$ M. A. Yandell, S. B. King, and D. M. Neumark, J. Chem. Phys. 140, 184317 (2014).

${ }^{28}$ A. Kunin, W.-L. Li, and D. M. Neumark, Phys. Chem. Chem. Phys. 18, 33226 (2016).

${ }^{29}$ E. Matthews, R. Cercola, G. Mensa-Bonsu, D. M. Neumark, and C. E. H. Dessent, J. Chem. Phys. 148, 084304 (2018).

${ }^{30}$ R. Cercola, E. Matthews, and C. E. H. Dessent, Mol. Phys., 1 (2019).

${ }^{31}$ A. Kunin, W.-L. Li, and D. M. Neumark, J. Chem. Phys. 149, 084301 (2018).

${ }^{32}$ I. Kulakowska, M. Geller, B. Lesyng, and K. L. Wierzchowski, Biochim. Biophys. Acta, Nucleic Acids Protein Synth. 361, 119 (1974).

${ }^{33}$ C. Desfrançois, H. Abdoul-Carime, and J.-P. Schermann, Int. J. Mod. Phys. B 10, 1339 (1996).

${ }^{34}$ J. H. Hendricks, S. A. Lyapustina, H. L. de Clercq, J. T. Snodgrass, and K. H. Bowen, J. Chem. Phys. 104, 7788 (1996).

${ }^{35}$ S. Carles, F. Lecomte, J. P. Schermann, and C. Desfrançois, J. Phys. Chem. A 104, 10662 (2000).

${ }^{36}$ M. Hanus, M. Kabelac, J. Rejnek, F. Ryjacek, and P. Hobza, J. Phys. Chem. B 108, 2087 (2004).

${ }^{37}$ R. A. Bachorz, W. Klopper, M. Gutowski, X. Li, and K. H. Bowen, J. Chem. Phys. 129, 054309 (2008).

38 J. Simons, J. Am. Chem. Soc. 103, 3971 (1981).

${ }^{39}$ P. K. Acharya, R. A. Kendall, and J. Simons, J. Am. Chem. Soc. 106, 3402 (1984). 
${ }^{40}$ B. Baguenard, J. C. Pinaré, F. Lépine, C. Bordas, and M. Broyer, Chem. Phys. Lett. 352, 147 (2002).

${ }^{41}$ C. Desfrancois, H. AbdoulCarime, and J. P. Schermann, J. Chem. Phys. 104, 7792 (1996).

${ }^{42}$ O. Dolgounitcheva, V. G. Zakrzewski, and J. V. Ortiz, Chem. Phys. Lett. 307, 220 (1999).

${ }^{43}$ O. Dolgounitcheva, V. G. Zakrzewski, and J. V. Ortiz, J. Phys. Chem. A 105, 8782 (2001).

${ }^{44}$ R. A. Bachorz, W. Klopper, and M. Gutowski, J. Chem. Phys. 126, 085101 (2007).

${ }^{45}$ R. J. Peláez, C. Blondel, C. Delsart, and C. Drag, J. Phys. B-At. Mol. Opt. 42, 125001 (2009).

${ }^{46}$ A. V. Davis, R. Wester, A. E. Bragg, and D. M. Neumark, J. Chem. Phys. 118, 999 (2003).

${ }^{47}$ A. E. Bragg, J. R. R. Verlet, A. Kammrath, O. Cheshnovsky, and D. M. Neumark, J. Am. Chem. Soc. 127, $15283(2005)$.

${ }^{48}$ W. C. Wiley, and I. H. McLaren, Rev. Sci. Instrum. 26, 1150 (1955).

${ }^{49}$ A. Eppink, and D. H. Parker, Rev. Sci. Instrum. 68, 3477 (1997).

${ }^{50}$ V. Dribinski, A. Ossadtchi, V. Mandelshtam, and H. Reisler, Rev. Sci. Instrum. 73, 2634 (2002).

${ }^{51}$ R. M. Young, M. A. Yandell, and D. M. Neumark, J. Chem. Phys. 134, 124311 (2011).

${ }^{52}$ G. Markovich, R. Giniger, M. Levin, and O. Cheshnovsky, J. Chem. Phys. 95, 9416 (1991).

${ }^{53}$ G. Markovich, S. Pollack, R. Giniger, and O. Cheshnovsky, J. Chem. Phys. 101, 9344 (1994). 
Table I. Lifetimes for the VB anion, autodetachment feature, and $\mathrm{I}^{-}$feature for $4.77 \mathrm{eV}$ pump $\mathrm{I}^{-} \cdot \mathrm{U} \cdot \mathrm{H}_{2} \mathrm{O}$ and comparison to previous $\mathrm{I}^{-} \cdot \mathrm{U}$ studies. The $\mathrm{I}^{-} \cdot \mathrm{U}$ data for the $\mathrm{VB}$ anion and autodetachment dynamics are from Ref. ${ }^{20}$, and for the $\mathrm{I}^{-}$feature the data is from Ref. ${ }^{25}$.

\begin{tabular}{|l|l|l|l|}
\hline \multicolumn{5}{|c|}{ VB anion } \\
\hline Cluster & $\mathrm{h} v_{\text {pump }}$ & $\tau_{\text {decay, },}$ & $\tau_{\text {decay, }, 2}$ \\
\hline $\mathrm{I}^{-} \cdot \mathrm{U} \cdot \mathrm{H}_{2} \mathrm{O}$ & $4.77 \mathrm{eV}$ & $540 \pm 240 \mathrm{fs}$ & $220 \pm 70 \mathrm{ps}$ \\
\hline $\mathrm{I}^{-} \cdot \mathrm{U}$ & $4.79 \mathrm{eV}$ & $390 \pm 80 \mathrm{fs}$ & $37 \pm 20 \mathrm{ps}$ \\
\hline \multicolumn{4}{|c|}{ Autodetachment } \\
\hline Cluster & $\mathrm{h} v_{\text {pump }}$ & $\tau_{\text {rise }}$ & $\tau_{\text {decay }}$ \\
\hline $\mathrm{I}^{-} \cdot \mathrm{U} \cdot \mathrm{H}_{2} \mathrm{O}$ & $4.77 \mathrm{eV}$ & $11.3 \pm 2.2 \mathrm{ps}$ & $285 \pm 70 \mathrm{ps}$ \\
\hline $\mathrm{I}^{-} \cdot \mathrm{U}$ & $4.79 \mathrm{eV}$ & $\sim 5 \mathrm{ps}$ & $\sim 50 \mathrm{ps}$ \\
\hline \multicolumn{4}{|c|}{$\mathrm{I}^{-}$} \\
\hline Cluster & $\mathrm{h} v_{\text {pump }}$ & $\tau_{\text {rise, }}$ \\
\hline $\mathrm{I}^{-} \cdot \mathrm{U} \cdot \mathrm{H}_{2} \mathrm{O}$ & $4.77 \mathrm{eV}$ & $32.5 \pm 2.6 \mathrm{ps}$ & $230 \pm 20 \mathrm{ps}$ \\
\hline $\mathrm{I}^{-} \cdot \mathrm{U}$ & $4.72 \mathrm{eV}$ & $36 \pm 3 \mathrm{ps}$ & $\tau_{\text {rise, }}$ \\
\hline
\end{tabular}




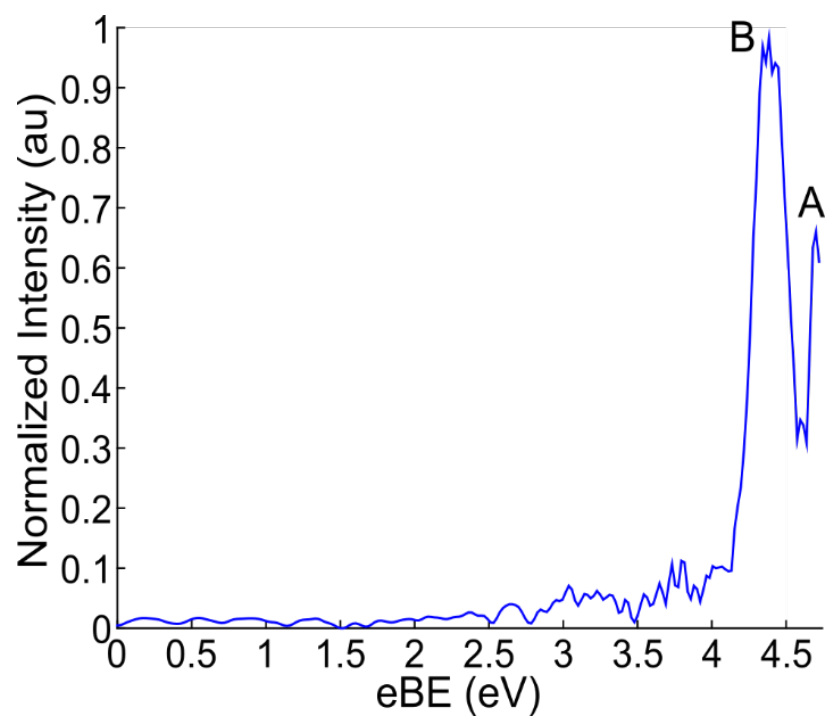

Figure 1. Single-photon photoelectron spectrum of $\mathrm{I}^{-} \cdot \mathrm{U} \cdot \mathrm{H}_{2} \mathrm{O}$ at $4.74 \mathrm{eV}$. This spectrum is reproduced from Kunin et al., J. Chem. Phys., 2018, 149, 084301, with the permission of AIP Publishing.

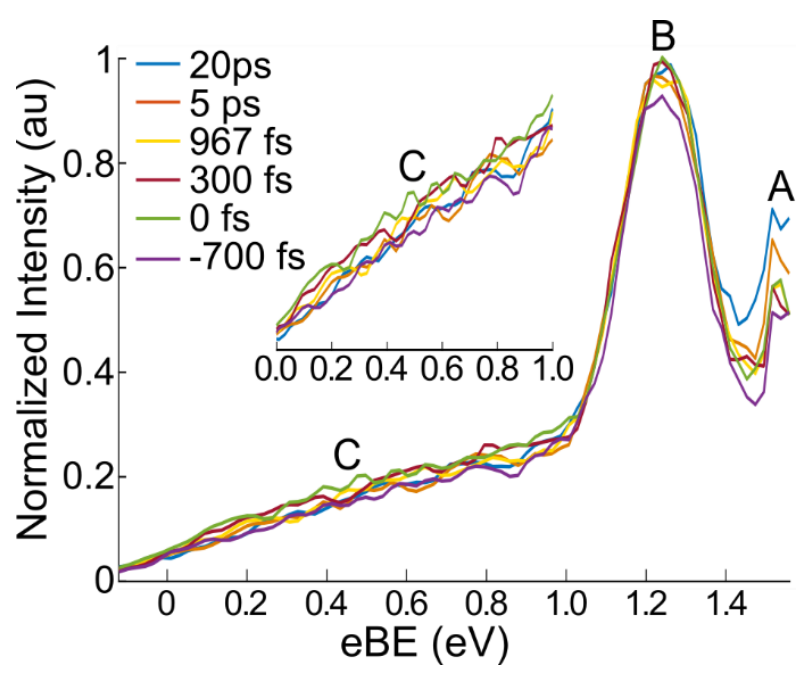

Figure 2. Photoelectron spectra of $\mathrm{I}^{-} \cdot \mathrm{U} \cdot \mathrm{H}_{2} \mathrm{O}$ at $4.77 \mathrm{eV}$ pump excitation energy and $1.58 \mathrm{eV}$ probe energy at selected delay times. 


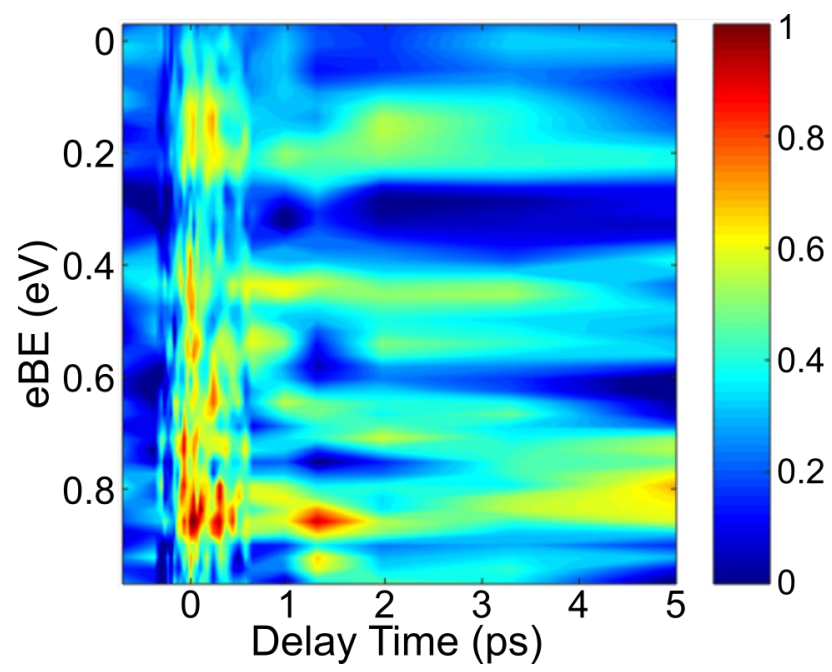

Figure 3. Representative background-subtracted time-resolved photoelectron spectra for feature $\mathrm{C}$ at short pump-probe delays for $\mathrm{I}^{-} \cdot \mathrm{U} \cdot \mathrm{H}_{2} \mathrm{O}$ at $4.77 \mathrm{eV}$ pump excitation energy and $1.58 \mathrm{eV}$ probe energy.

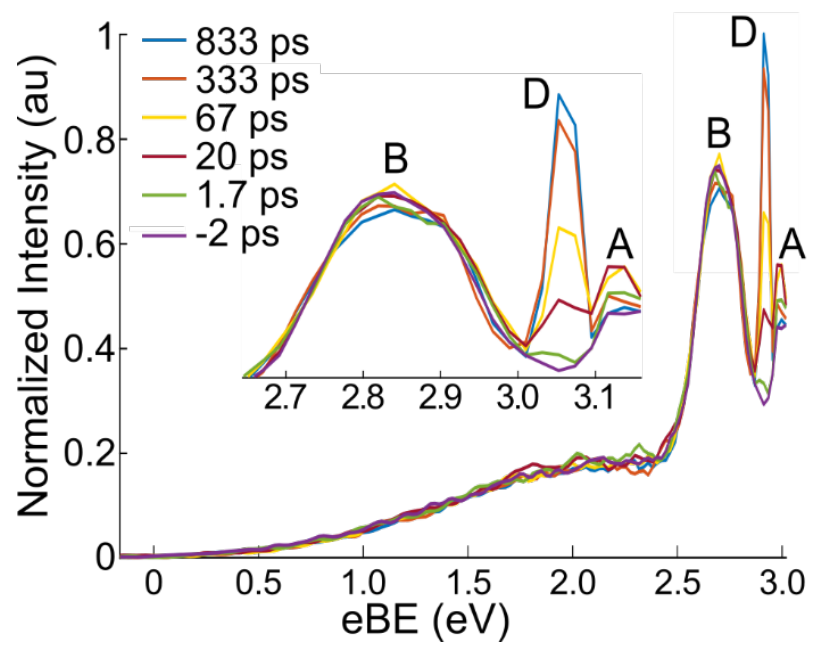

Figure 4. Photoelectron spectra of $\mathrm{I}^{-} \cdot \mathrm{U} \cdot \mathrm{H}_{2} \mathrm{O}$ at $4.77 \mathrm{eV}$ pump excitation energy and $3.18 \mathrm{eV}$ probe energy at selected delay times. 


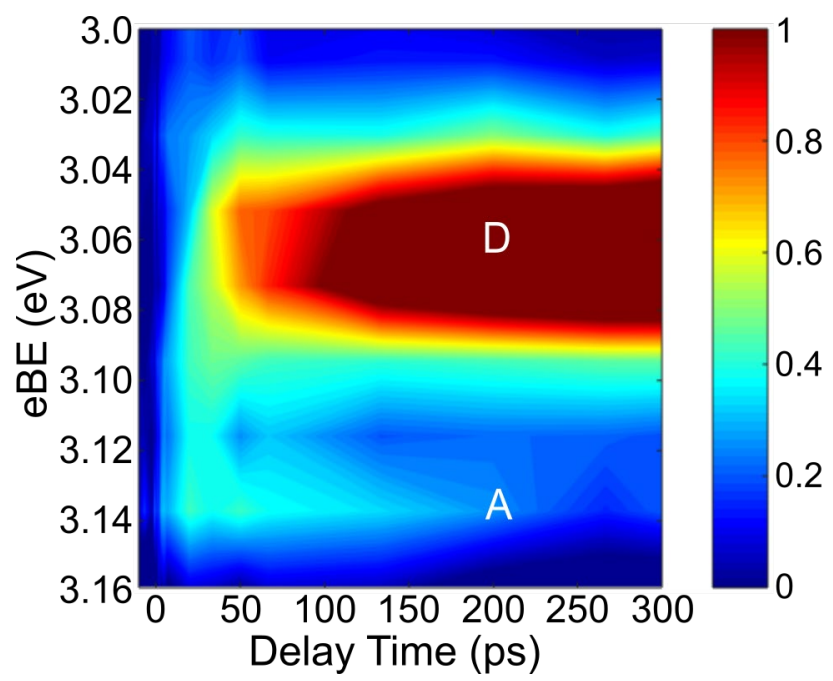

Figure 5. Representative background-subtracted time-resolved photoelectron spectra for features A (near maximum eBE, eKE $\sim 0-0.07 \mathrm{eV})$, and $\mathrm{D}(\mathrm{eBE}=3.06 \mathrm{eV})$ for $\mathrm{I}^{-} \cdot \mathrm{U} \cdot \mathrm{H}_{2} \mathrm{O}$ at $4.77 \mathrm{eV}$ pump excitation energy and $3.18 \mathrm{eV}$ probe energy.
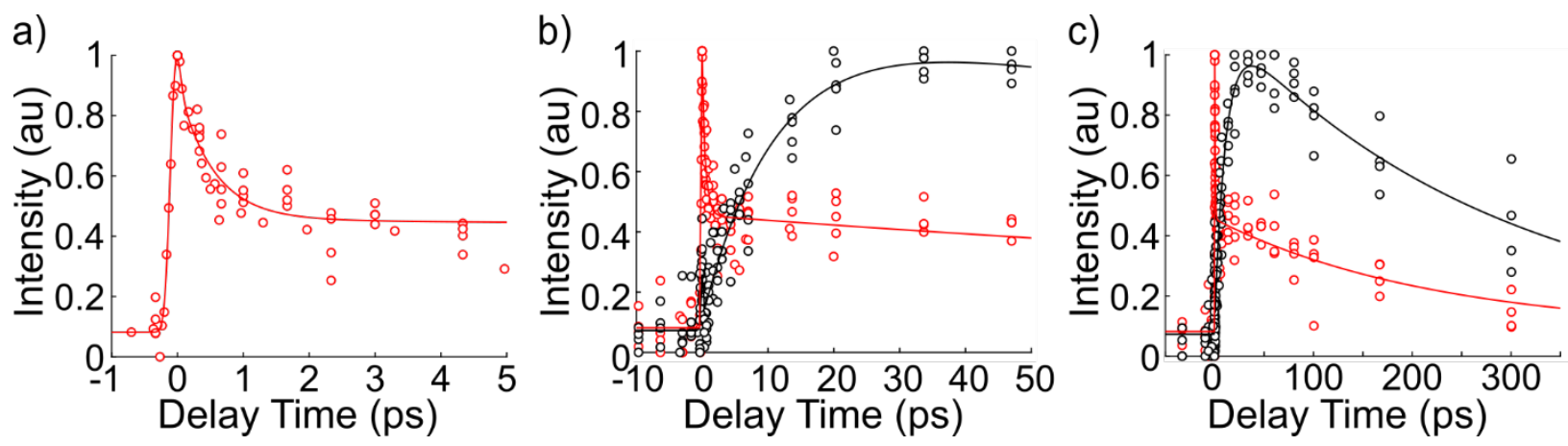

Figure 6. Concatenated normalized integrated intensities for features A (black, autodetachment) and $\mathrm{C}$ (red, VB anion) at a) early time delays, b) 10s of ps, and c) long time delays from excitation at $4.77 \mathrm{eV}$ and probed with $1.58 \mathrm{eV}$. Feature A rises in $11.3 \pm 2.2 \mathrm{ps}$ and decays in 285 $\pm 70 \mathrm{ps}$. The rise time for feature $\mathrm{C}$ is cross-correlation limited and the decay is $540 \pm 240$ fs and $220 \pm 70$ ps. 

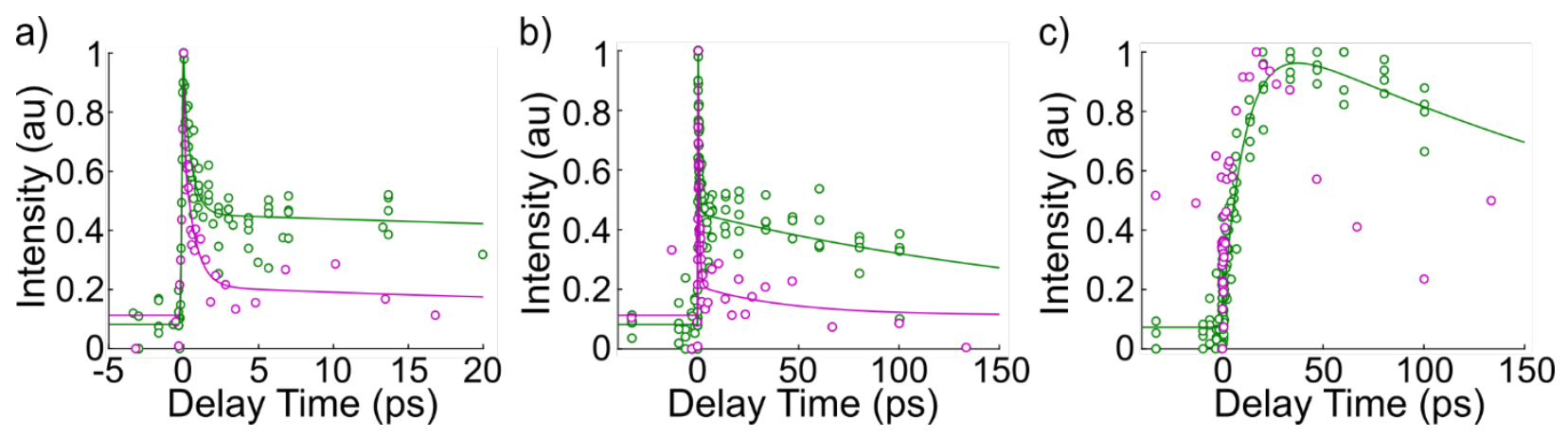

Figure 7. Comparison of concatenated normalized integrated intensities for a) the VB anion at early times and b) long times and c) autodetachment dynamics for $\mathrm{I}^{-} \cdot \mathrm{U} \cdot \mathrm{H}_{2} \mathrm{O}$ (green) at $4.77 \mathrm{eV}$ pump excitation energy and $1.58 \mathrm{eV}$ probe energy and $\mathrm{I}^{-} \cdot \mathrm{U}$ (purple) at $4.79 \mathrm{eV}$ pump excitation energy and $1.57 \mathrm{eV}$ probe energy.

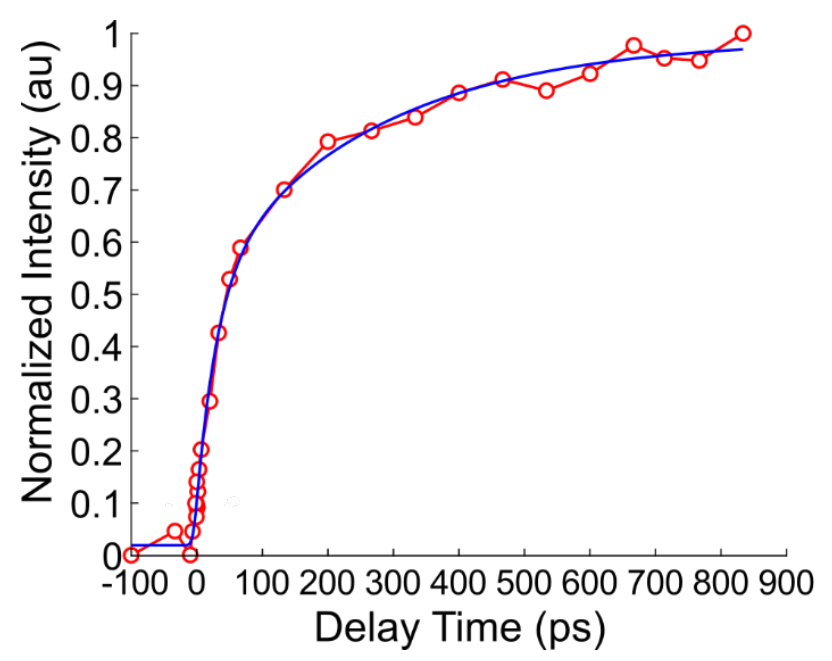

Figure 8. Normalized integrated intensity for feature $\mathrm{D}$ at $4.77 \mathrm{eV}$ pump excitation energy and $3.18 \mathrm{eV}$ probe energy. Feature $\mathrm{D}$ rises bi-exponentially with time constants of $32.5 \pm 2.6 \mathrm{ps}$ and $226 \pm 20$ ps. 